\title{
Time dependence of NMR observables reveals salient differences in the accumulation of early aggregated species between human islet amyloid polypeptide and amyloid- $\beta \dagger$
}

\author{
Anaïs R. F. Hoffmann, Lucie Caillon, Lilian Shadai Salazar Vazquez, \\ Pierre-Alexandre Spath, Ludovic Carlier, Lucie Khemtémourian (D) * and \\ Olivier Lequin (D)*
}

\begin{abstract}
Type 2 diabetes mellitus and Alzheimer's disease are characterized by the accumulation of fibrillar amyloid deposits consisting mainly of islet amyloid polypeptide (IAPP) and amyloid- $\beta$ (A $\beta$ ), respectively. Fibril formation is a multi-step nucleation process that involves the transient build-up of oligomeric species that are thought to be the most toxic components. To gain more insight into the molecular mechanism of early IAPP aggregated species formation, we performed a combination of direct and indirect biophysical approaches on IAPP and also on A 342 for the sake of comparison. Thioflavin T fluorescence kinetics measurements revealed a stronger autocatalytic behaviour of IAPP and a weaker concentration dependence of fibrillization half-time $t_{1 / 2}$, as compared to A $\beta 42$. Our NMR experiments highlight the absence of micelle reservoir or supercritical regime in the studied concentration range, indicating that the low concentration dependence of IAPP fibril formation can be ascribed to saturable pathways. IAPP and A 442 displayed marked differences in formation of oligomeric species, as observed by $1 D{ }^{1} \mathrm{H}$, pulsed-field gradient (PFG) diffusion and saturation transfer difference (STD) NMR experiments. A fast equilibrium between monomer and oligomeric species was detected in the case of A $\beta 42$ but not IAPP, with a significant build-up of aggregated species, as shown by the time dependence of diffusion coefficient and STD magnetization transfer efficiency during the aggregation process. Altogether our data show significant differences between IAPP and A 342 regarding the microscopic events of amyloid species formation.
\end{abstract}

\section{Introduction}

The aggregation of misfolded proteins into insoluble fibrils is a characteristic of more than 20 human amyloid diseases, including Alzheimer's disease, Hungtinton's disease, Parkinson's disease, prion diseases and type 2 diabetes mellitus (T2DM). ${ }^{1,2}$ T2DM is characterized metabolically by hyperglycemia associated with insulin resistance and a relative defect in insulin production. Histologically, the presence of fibrillar amyloid deposits is observed in the pancreatic islets of Langerhans of T2DM patients. ${ }^{3,4}$ Human islet amyloid polypeptide (IAPP), a 37 amino

Sorbonne Université, Ecole Normale Supérieure, PSL University, CNRS, Laboratoire des Biomolécules (LBM), 4 place Jussieu, F-75005 Paris, France. E-mail: lucie.khemtemourian@upmc.fr, olivier.lequin@upmc.fr;

Tel: +33144273113

$\dagger$ Electronic supplementary information (ESI) available: Fig. S1 Influence of TROL probe on IAPP fibril formation. Fig. S2 Influence of temperature on STD signal intensity of A $\beta 42$. Fig. S3 Silver-stained polyacrylamide gels of IAPP and A $\beta 42$ samples after different incubation times. See DOI: $10.1039 / \mathrm{c} 7 \mathrm{cp} 07516 \mathrm{~b}$ acid peptide also known as amylin, is the major constituent of these amyloid deposits. Under normal conditions, IAPP peptide, which is co-secreted with insulin, remains soluble but, in the pancreas of T2DM patients, the increase in peptide concentration and misfolding lead to oligomerization and amyloid fibrils formation at the surface of cells, associated with cell toxicity. ${ }^{5,6}$

The aggregation of amyloid proteins follows a nucleationdependent polymerization process, characterized by a well-defined sigmoidal transition. ${ }^{1}$ After an initial lag phase in which monomers, and possibly oligomers, dominate the population, a growth phase is observed during which fibrils elongate and reach a final plateau state. ${ }^{7}$ It is now established that aggregates can be formed not only during initial nucleation in solution but also through secondary processes involving pre-existing fibrils. In that latter case, fibrils can directly catalyze the formation of the aggregates at their surface or can fragment into smaller fibrils providing new elongation templates.

One of the most widely studied amyloid diseases is Alzheimer's disease and $\mathrm{A} \beta 42$, the main $\mathrm{A} \beta$ amyloid form involved, is commonly 
used as a model for amyloid fibril formation studies. ${ }^{8}$ The kinetics of $\mathrm{A} \beta 42$ fibrillization have been extensively studied by several groups to characterize nucleation pathway mechanisms and derive mathematical models of aggregation. Relevant mechanistic information can be obtained by studying the dependence on monomer concentration of kinetic parameters, in particular the half-time $t_{1 / 2}$ of fibril formation. ${ }^{8}$ Based on kinetics studies, A $\beta 42$ was shown to fibrillate mainly through fibril-catalysed secondary process rather than direct primary nucleation pathway. ${ }^{9}$ The shorter A $\beta 40$ peptide exhibited different mechanistic behaviours with a saturable secondary nucleation, reflecting a more complex multistep nature of molecular aggregation at the fibril surface. ${ }^{10}$

Characterizing low molecular weight aggregated species that form during the aggregation process is important not only to decipher the molecular basis of nucleation pathways but also to address amyloid toxicity. ${ }^{11,12}$ Indeed, increasing evidence has shown that the toxicity of amyloid peptides is more likely mediated by oligomeric species ${ }^{13-15}$ or by the ongoing fibrillization process rather than by the fibrils themselves. ${ }^{16,17}$ It is now well established that the toxicity of $\mathrm{A} \beta$ is mediated through toxic oligomers that are distinct from the insoluble amyloid fibrils. Many off- and onpathway oligomeric intermediates of $\mathrm{A} \beta$ have been isolated, both in vitro and in situ from tissue samples of Alzheimer's patients. ${ }^{18,19}$ Small soluble oligomers, such as dimers, trimers, tetramers and higher oligomers have been observed and isolated for $\mathrm{A} \beta .^{20-24} \mathrm{~A}$ recent study has proposed trimeric and tetrameric species as the most toxic low order oligomers of $A \beta .^{25}$

Although they do not seem to be evolutionary related, IAPP and $A \beta 42$ share about $40 \%$ amino acid sequence similarity and form $\beta$-sheet-rich amyloid fibrils exhibiting similar morphologies. It was thus proposed that IAPP and A $\beta 42$ could have common mechanisms of amyloid fibril formation and assume similar mechanisms of toxicity. ${ }^{11,26}$ Furthermore, IAPP and A $\beta 42$ were experimentally shown to cross-seed fibrillization and form hybrid amyloid fibers. ${ }^{27}$ However, the existence of IAPP small oligomeric intermediates is more controversial and available data from the literature demonstrating the existence of IAPP oligomeric species are scarce. The group of Glabe suggested that synthetic IAPP oligomers cause cellular leakage on SH-SY5Y cells. ${ }^{28}$ Nevertheless, the size of the IAPP oligomers was not precisely determined as was done with $\mathrm{A} \beta$. Janson et al., using light-scattering techniques, demonstrated that cell membrane disruption is caused by intermediate-sized toxic amyloid particles containing 25 to 500 monomeric IAPP. ${ }^{29}$ Later, large spherical aggregates were observed by immunomicroscopy in both diabetic and nondiabetic human pancreas, with an increase in population and size found in diabetic islets. ${ }^{30}$ However such aggregates have not been clearly associated with amyloid toxicity. Attempts to characterize small soluble oligomers in vitro of IAPP have been less successful. An ultracentrifugation study failed to detect small oligomeric IAPP which led the authors to suggest that the putative IAPP toxic oligomers should contain more than 100 monomers. ${ }^{31}$ Furthermore, using pulsed field gradient NMR spectroscopy, Soong et al. observed only the presence of large oligomeric IAPP (oligomers greater than $100 \mathrm{~nm}$ ) and did not succeed to observe small oligomeric IAPP. ${ }^{32}$ Finally, another study using ${ }^{19}$ F NMR revealed that IAPP form fibrils without significant build-up of nonfibrillar intermediates. ${ }^{33}$ More recently, small soluble oligomers, from dimers up to hexamers, could be detected by Ion Mobility Spectrometry-Mass Spectrometry (IMS-MS). ${ }^{34}$ However it remains difficult to address whether these oligomers correspond to on-pathway or off-pathway species as they are observed for both human amyloidogenic and rat non-amyloidogenic IAPP species. Finally, two-dimensional infrared spectroscopy was the only biophysical technique that was able to detect and characterize a transient $\beta$-sheet population at high IAPP concentration. ${ }^{35}$ This intermediate was predicted to contain at least 5 monomers and has a parallel $\beta$-sheet organisation that differs from the structure of final fibrils, requiring a structural rearrangement during polymerization. ${ }^{36}$

In this paper, we wanted to dissect the molecular events of early IAPP aggregation. For that purpose we have conducted a comprehensive set of biophysical studies on IAPP, based on fluorescence, circular dichroism (CD), transmission electron microscopy (TEM), liquid-state NMR and gel electrophoresis. These experiments were carried out under similar experimental conditions, in order to draw meaningful comparison between the different techniques. For the sake of comparison, these techniques were also applied on $A \beta 42$, which is one of the most characterized amyloid peptides. Our data show that IAPP exhibits significant differences with $A \beta 42$ in terms of kinetics of fibril formation, presence of oligomers observed either directly or indirectly, and detection of momoner/oligomer equilibria.

\section{Experimental}

\subsection{Peptide synthesis}

Human IAPP (comprising an amidated C-terminus and a $\mathrm{Cys}^{2}{ }^{2} \mathrm{Cys}^{7}$ disulfide bridge) and human $\mathrm{A} \beta_{1-42}$ (referred herein as $A \beta 42$ ) were synthesized by Fmoc chemistry at the Institut de Biologie Intégrative (IFR83-Université Pierre et Marie Curie). Peptides were purified by reverse phase high-performance liquid chromatography (HPLC). Peptides purity was higher than 95\%, as determined by analytical HPLC, and their identity was confirmed by MALDI-TOF mass spectrometry.

\subsection{Sample preparation}

A critical requirement for measuring aggregation kinetics of amyloid peptides with good reproducibility is to prepare peptide samples that are largely monomeric and free of aggregates. Peptide stock solutions were freshly prepared prior to all experiments, using the same batch. In the case of IAPP, lyophilized peptide was solubilized at a concentration of $1 \mathrm{mM}$ in hexafluoroisopropanol (HFIP) and incubated for one hour. Then HFIP was evaporated under a nitrogen stream and the sample was dried by vacuum dessication for at least $30 \mathrm{~min} .{ }^{37,38}$ The resulting peptide film was solubilized at a concentration of $1 \mathrm{mM}$ in DMSO for fluorescence experiments or directly in $10 \mathrm{mM}$ sodium phosphate buffer, $\mathrm{pH} 7.4$, in other experiments. The peptide concentration range was $5-75 \mu \mathrm{M}$. In the case of $\mathrm{A} \beta 42$, peptide stock solutions were prepared by solubilizing the peptide at a 
concentration of $1 \mathrm{mM}$ in a $1 \% \mathrm{NH}_{4} \mathrm{OH}$ aqueous solution, as previously described. ${ }^{39}$ This preparation protocol was shown to produce seed free peptide solutions ${ }^{40}$ and ensured good experimental reproducibility. A $\beta 42$ peptides samples were prepared in $10 \mathrm{mM}$ sodium phosphate buffer, $\mathrm{pH}$ 7.4, for circular dichroism, electron microscopy and gel electrophoresis experiments. The same experimental conditions were applied for NMR experiments except that the buffer was prepared in $100 \% \mathrm{D}_{2} \mathrm{O}$. A $\beta 42$ and IAPP seeds were prepared from peptide samples incubated several days at room temperature. Samples were sonicated for $2 \mathrm{~min}$ in a bath sonicator (Branson model 2510) to obtain monodispersed fibril fragments. ${ }^{16}$

\subsection{Fluorescence assays}

The kinetics of fibril formation was monitored using the fluorescence intensity variation of thioflavin $\mathrm{T}$ (ThT) or tryptophanol (TROL). Fluorescence measurements were taken on a Fluostar Optima fluorimeter (BMG LabTech, Germany) using standard 96 well flat-bottom black microtiter plates and excitation/emission filters of 440/480 $\mathrm{nm}$ for ThT and 280/340 nm for TROL. Prior to the first measurements, the plates were shaken at $600 \mathrm{rpm}$ for $10 \mathrm{~s}$. The fluorescence was measured at room temperature from the top of the plates every 10 minutes, without any agitation.

The fluorescence assays were prepared in $200 \mu \mathrm{L}$ wells in $10 \mathrm{mM}$ sodium phosphate $\mathrm{pH} 7.4$ buffer containing 5 to $75 \mu \mathrm{M}$ peptide and equimolar amount of ThT or TROL. The assays were performed 3 times, each in triplicate, on different days, using different IAPP stock solutions. The replicates of each system showed consistent reproducibility.

The ThT fluorescence curves were analyzed using R program and fitted to a Boltzmann sigmoidal equation (eqn (1)), where $F_{\mathrm{i}}$ and $F_{\mathrm{f}}$ are the initial and final fluorescence values. The sigmoid equation was modified to include a slope in the final fluorescence plateau. ${ }^{41}$

$$
F=\frac{F_{\mathrm{i}}-\left(F_{\mathrm{f}}+a t\right)}{1+\mathrm{e}^{k\left(t-t_{1 / 2}\right)}}+\left(F_{\mathrm{f}}+a t\right)
$$

Two kinetic parameters were estimated: the half-time $t_{1 / 2}$, corresponding to the time for which the fluorescence reaches $50 \%$ of its maximal intensity, and the rate of elongation $k$.

\subsection{Circular dichroism (CD)}

The changes in secondary structure of peptides alone or in the presence of ThT or TROL fluorescent dye (1:1 molar ratio) were measured using a Jasco J-815 CD spectropolarimeter with a Peltier temperature-controlled cell holder over the wavelength range 190-260 $\mathrm{nm}$. Measurements were carried out in $10 \mathrm{mM}$ sodium phosphate buffer, pH 7.4 at $30{ }^{\circ} \mathrm{C}$, using quartz cells of $1 \mathrm{~mm}$ path length. For each sample, measurements were taken every $0.2 \mathrm{~nm}$ at a scan rate of $10 \mathrm{~nm} \mathrm{~min}{ }^{-1}$. Spectra were acquired every 30 minutes over a period of 7 to 48 hours. Peptide concentration was $75 \mu \mathrm{M}$.

\subsection{Transmission electron microscopy (TEM)}

TEM was performed on samples after different times of incubation, at Faculté de Médecine Xavier Bichat or Institut de Biologie Paris Seine, in order to analyze aggregates and fibrils morphology.
$25 \mu \mathrm{L}$ of each sample (75 $\mu \mathrm{M}$ peptide concentration) were adsorbed onto glow-discharged carbon coated 200 mesh copper grids. After the grids have dried off, the grids were negatively stained during $45 \mathrm{~s}$ with a $2.5 \%$ uranyl acetate solution in 1:1 ethanol/water. The grids were then blotted and dried. Grids were examined using JEOL electron microscopes operating at $80 \mathrm{kV}$ or $200 \mathrm{kV}$. The size of fibrils was determined using Image software.

\subsection{NMR spectroscopy}

NMR experiments were recorded on a Bruker Avance III spectrometer operating at a ${ }^{1} \mathrm{H}$ frequency of $500 \mathrm{MHz}$ and equipped with a TCI cryoprobe. Experiments were processed and analyzed with TopSpin program (Bruker). Peptides samples were prepared at a concentration of $75 \mu \mathrm{M}$ in $10 \mathrm{mM}$ sodium phosphate buffer, $\mathrm{pH} 7.4$, in $\mathrm{D}_{2} \mathrm{O}$. NMR Shigemi tubes of $5 \mathrm{~mm}$ diameter were used, comprising a sample volume of $280 \mu \mathrm{L}$. One-dimensional ${ }^{1} \mathrm{H}$ spectra were acquired with an acquisition time of $0.6 \mathrm{~s}$, a relaxation delay of $2 \mathrm{~s}$, and 512 scans, corresponding to an experimental time of about $22 \mathrm{~min}$. The residual solvent HOD resonance was suppressed by weak presaturation during the relaxation delay.

Diffusion coefficients $D_{\mathrm{t}}$ were measured using a stimulated echo sequence ${ }^{42}$ with bipolar squared gradient pulses of constant duration (6 ms) and variable gradient amplitude along the longitudinal axis (linear ramp of 30 points, from 0.03 to $0.44 \mathrm{~T} \mathrm{~m}^{-1}$ ). The diffusion delay was $0.15 \mathrm{~s}$. The pulsed field gradients were calibrated on a $\mathrm{D}_{2} \mathrm{O}$ sample using a diffusion coefficient value for HOD of $1.9 \times 10^{-9} \mathrm{~m}^{2} \mathrm{~s}^{-1}$ at $25^{\circ} \mathrm{C} .{ }^{43}$ Experiments were processed and analyzed with NMRPipe software. The hydrodynamic radius $R_{\mathrm{H}}$ was calculated from Stokes-Einstein equation $D_{\mathrm{t}}=k_{\mathrm{B}} T / 6 \pi \eta R_{\mathrm{H}}$ where $k_{\mathrm{B}}$ is Boltzmann's constant, $T$ is the absolute temperature and $\eta$ is the viscosity. $\mathrm{D}_{2} \mathrm{O}$ solvent viscosities at different temperatures were taken from published values. ${ }^{44}$

1D ${ }^{1} \mathrm{H}$ STD experiments were acquired with 320 scans (total duration of $50 \mathrm{~min}$ ). A train of Gaussian shaped pulses (50 ms pulse length, $0.1 \mathrm{kHz} B_{1}$ field, total duration of $3 \mathrm{~s}$ ), applied alternatively on resonance $(-0.5 \mathrm{ppm})$ and off resonance (+30 ppm), as described. ${ }^{39,45}$

\subsection{Gel electrophoresis}

Peptide samples were analysed by PAGE, using Tris-glycine gels with $4-20 \%$ acrylamide gradient (Mini-PROTEAN TGX, Biorad). Running buffer contained $25 \mathrm{mM}$ Tris, $18.75 \mathrm{mM}$ glycine and $0.001 \%$ SDS. Samples were diluted in one volume of loading buffer (125 mM Tris- $\mathrm{HCl}$ ) containing 0.05\% bromophenol blue, $10 \%(\mathrm{v} / \mathrm{v})$ glycerol and $4 \%(\mathrm{w} / \mathrm{v})$ SDS, without heating. After migration, bands were revealed by silver staining.

\section{Results}

\subsection{Fibril formation monitored by ThT and TROL fluorescence, CD spectroscopy and TEM}

The time course of the aggregation of human IAPP and A $\beta 42$ was first followed by ThT fluorescence, which is a widely used 
A

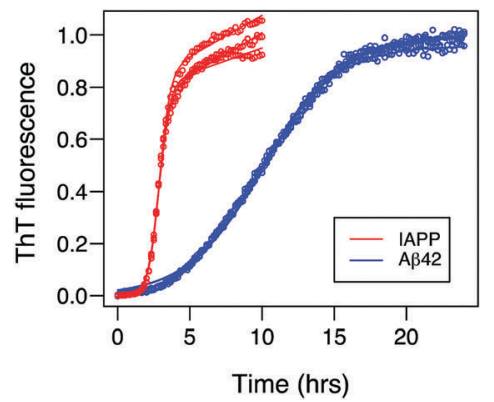

B

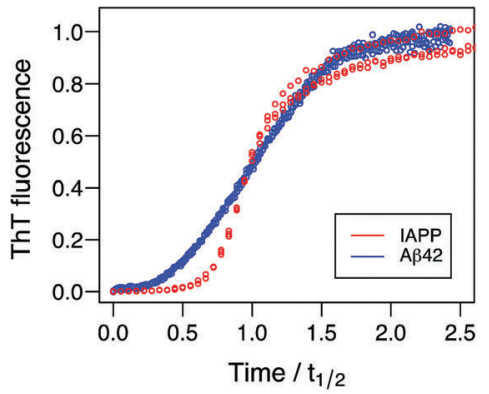

C

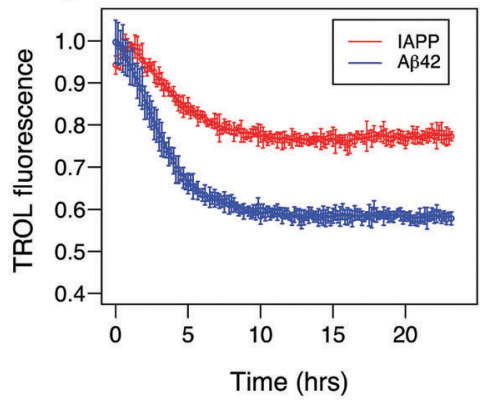

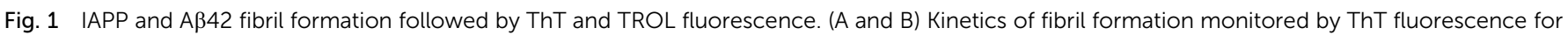

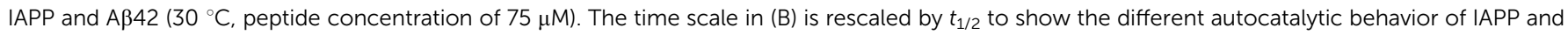
A $\beta 42$. (C) Evolution of TROL fluorescence for IAPP and A $\beta 42$.

method to monitor amyloid fibril formation. ${ }^{46}$ Indeed, the fluorescence of free ThT in solution is known to be largely quenched while it increases when ThT binds to amyloid fibrils (and also prefibrillar aggregates). ${ }^{47}$ Fig. 1A shows typical curves obtained for IAPP at a concentration of $75 \mu \mathrm{M}$ in $10 \mathrm{mM}$ phosphate buffer at $\mathrm{pH}$ 7.4. These kinetic data display the characteristic sigmoidal shape observed for amyloid peptides, comprising a short lag phase, a sharp sigmoidal transition and a final plateau. Fitting to a Boltzmann equation yields a half-time $t_{1 / 2}$ of $2.8 \pm 0.05 \mathrm{~h}$ and an elongation rate $k$ of $2.5 \pm 0.05 \mathrm{~h}^{-1}$.

To provide deeper insight into the formation of early IAPP aggregated species on the fibrillization pathway, we chose to use another fluorescent probe, tryptophanol (TROL). This molecule was described to bind to prefibrillar species, such as globular oligomers or short protofibrils, but not to mature fibrils. ${ }^{48}$ In contrast with ThT, the binding leads to quenching of TROL fluorescence. Fig. 1C shows an initial stationary state, which is followed by a $20 \%$ decrease of TROL fluorescence. These steps closely match the lag phase and the elongation phase observed in the evolution of ThT fluorescence. These data indicate that TROL probe is not able to detect the accumulation of small IAPP oligomeric species during the lag phase of fibril formation.

The CD spectra of freshly prepared IAPP sample under the same conditions, showed curve with a minimum at $200 \mathrm{~nm}$, indicating a prevailing unordered conformation (Fig. 2A). Then, after a few hours of incubation, IAPP adopts a $\beta$-sheet structure characterized by a negative minimum around $218 \mathrm{~nm}$. The observation of an isodichroic point near $208 \mathrm{~nm}$ is consistent with a two-state conformational transition between random coil and $\beta$-sheet species (such as early ordered oligomers or later aggregated species) during the aggregation process. The kinetics of IAPP fibril formation was also followed by TEM (Fig. 3). Globular aggregates are observed after 1 hour of aggregation (Fig. 3A). Short and long fibrils can be observed after 2 hours (not shown) and 5 hours, respectively (Fig. 3B). They coexist with globular aggregates (Fig. 3B), which are no longer observed at the end of incubation (Fig. 3C). Final samples exhibit typical amyloid fibril morphology with large and dense mats of fibrils that were about 5 to $8 \mathrm{~nm}$ wide.

As a comparison, $\mathrm{A} \beta 42$ fibrillization was studied under the same experimental conditions (buffer, temperature, peptide concentration) as IAPP. From a qualitative point of view, A $\beta 42$ peptide shows similar features as IAPP regarding the sigmoidal shape of ThT fluorescence curve (Fig. 1A), the conformational transition as observed by CD spectroscopy (Fig. 2B) and the morphology of formed fibrils as visualized by TEM (Fig. 3).

A quenching of TROL fluorescence is also observed in the time course of $\mathrm{A} \beta 42$ aggregation (Fig. 1C). The fluorescence decay profile differs from that observed with IAPP as no lag time is
A

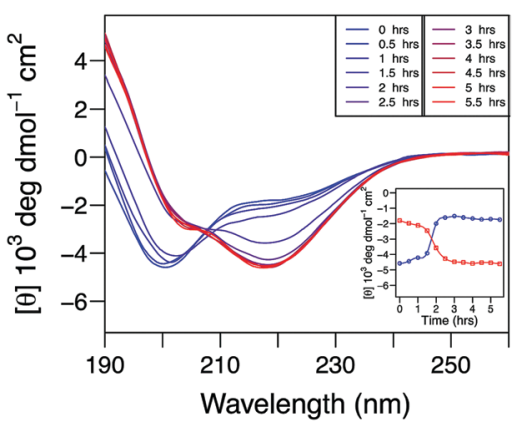

B

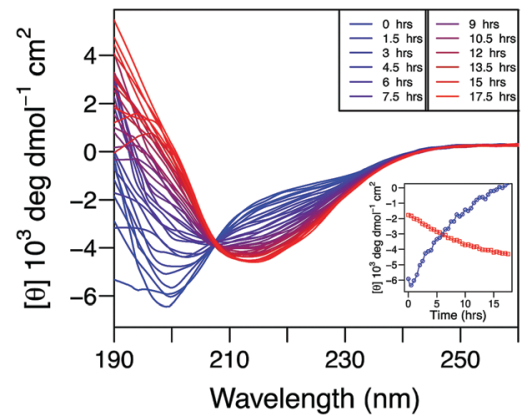

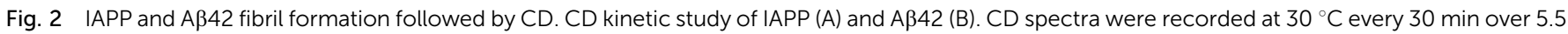

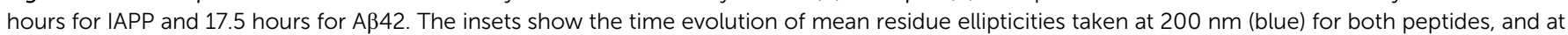
$218 \mathrm{~nm}$ or $214 \mathrm{~nm}$ (red) for IAPP and A 342 , respectively. 
A
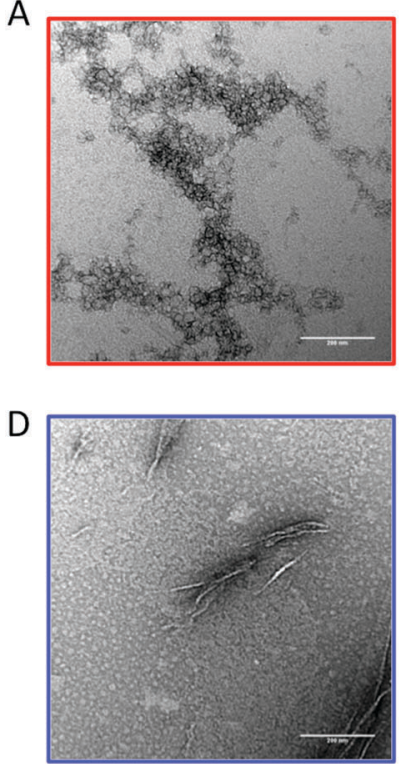

B

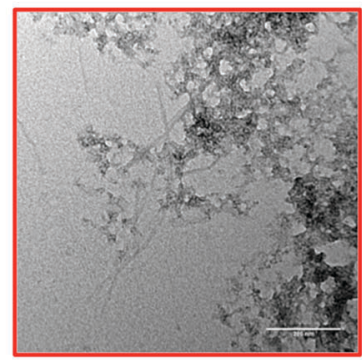

E

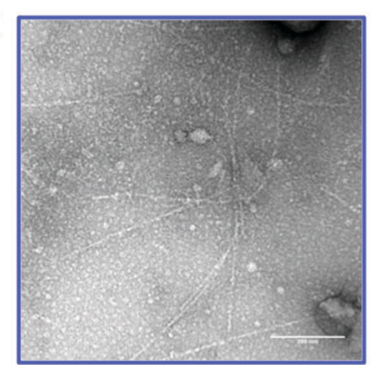

C

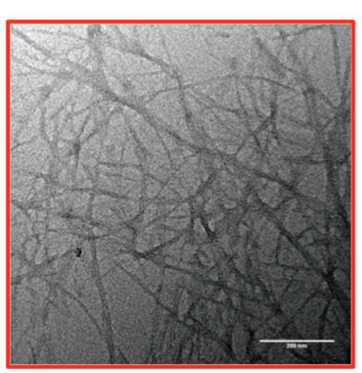

$\mathrm{F}$

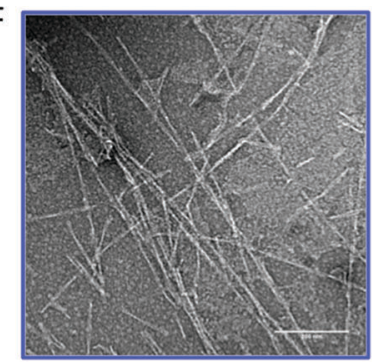

Fig. 3 IAPP and A 42 fibril formation followed by TEM. Negatively stained TEM images of IAPP (upper panels) and A 42 (lower panels) after 1 hour (A and C), 5 hours ( $B$ and $D$ ) and 3 days of incubation ( $C$ and $E$ ).

observed in the decay of TROL fluorescence and most of the fluorescence quenching $(40 \%)$ occurs during the ThT-defined lag phase. This indicates that prefibrillar species detectable with TROL (small or large oligomers) accumulate prior to the fibril elongation phase in the case of $A \beta 42$.

Another difference concerns the aggregation kinetics of $\mathrm{A} \beta 42$, which is much slower under identical experimental conditions, with a half-time $t_{1 / 2}$ of $9.8 \pm 0.15 \mathrm{~h}$ and an elongation rate $k$ of $0.35 \pm 0.01 \mathrm{~h}^{-1}$. The steeper sigmoidal transition of IAPP ThT curve indicates a higher cooperativity of the fibrillization process for IAPP. This can been shown by plotting normalized fibrillization kinetics on the same relative scale $t / t_{1 / 2}$ (Fig. 1B). Such behaviour can reflect mechanistic differences in the aggregation pathways of these two peptides. In particular, the stronger autocatalytic behaviour of IAPP relative to A $\beta 42$ may result from an increased contribution of secondary pathways such as fibril fragmentation or fibril-catalyzed nucleation.

\subsection{Concentration-dependence of the kinetics of IAPP fibril formation}

In order to get information on mechanistic aspects of IAPP fibrillization, we next examined the influence of peptide concentration on the kinetics of fibril formation (Fig. 4A). In particular we analyzed the effect of varying IAPP initial concentration on ThT plateau fluorescence and $t_{1 / 2}$ values. Experiments were conducted in the absence of agitation in order to minimize pathways involving fibril fragmentation, as described for $\mathrm{A} \beta 42$. The final ThT plateau fluorescence was found to vary linearly with initial peptide concentration (Fig. 4B). In contrast, we observe a weak dependence of $t_{1 / 2}$ on peptide concentration (5-75 $\mu \mathrm{M}$ range). By plotting $t_{1 / 2}$ as a function of concentration on a logarithmic scale, $t_{1 / 2}$ shows a concentration dependence as a power function $t_{1 / 2} \sim c^{\gamma}$, with an exponent $\gamma$ of -0.2 (Fig. 4C). The $\gamma$ exponent is too low with respect to theoretical values expected if primary nucleation step or secondary nucleation pathways predominate. It is closer to the value of -0.5 that would be obtained
A

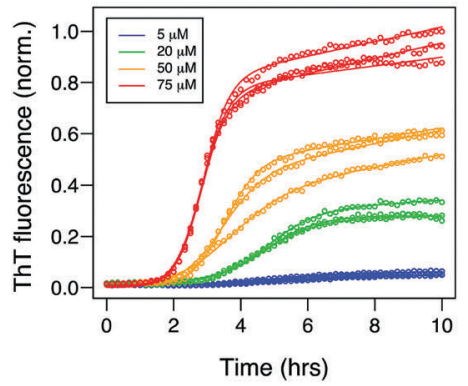

B

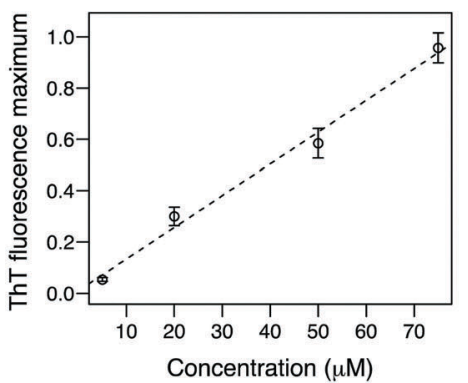

\section{C}

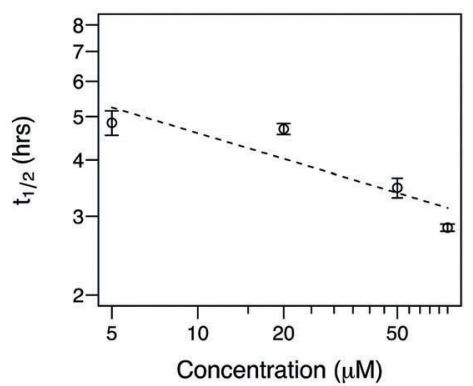

Fig. 4 Influence of IAPP concentration on the kinetics of fibril formation. (A) ThT fluorescence kinetics at $30{ }^{\circ} \mathrm{C}$. The fluorescence curves were fitted to a Boltzmann equation to extract the maximal intensity at the plateau and half-time $t_{1 / 2}$. (B) Plot of ThT fluorescence plateau intensity versus IAPP concentration. (C) Logarithmic plot of half-time $t_{1 / 2}$ versus IAPP concentration. 
in the case of fibril fragmentation processes, as was observed for $\mathrm{A} \beta 42$ when shear forces were applied by agitation. ${ }^{9}$ However a dominant contribution of such pathway is unlikely for IAPP in the absence of agitation. Several models have been proposed to account for a weak concentration dependence of $t_{1 / 2}$. The model described by Powers et al. involves an irreversible polymerization regime, in which oligomeric species turn to be more stable, and consequently more abundant, than the monomer beyond a supercritical concentration. ${ }^{49}$ Nevertheless, this model is not compatible with our NMR results showing at the highest concentration examined that (i) the monomer is the most abundant species at the beginning of the kinetics and (ii) no low molecular weight oligomers are detected (see below). Recently, the supercritical concentration of IAPP was estimated to be in the range 150-250 $\mu \mathrm{M}$, based on 2D IR studies. ${ }^{36}$ This indicates that our studied concentration range falls below the supercritical concentration and that the weak concentration dependence cannot be accounted for by a supercritical regime. Therefore such weak concentration dependence implies a saturation of microscopic processes at high monomer concentration, a saturable fibril elongation being most likely.

\subsection{Kinetics of monomer consumption followed by 1D ${ }^{1}$ H NMR spectroscopy}

We next performed liquid state ${ }^{1} \mathrm{H}$ NMR studies to follow the kinetics of depletion of monomer, and detect putative soluble low molecular weight species of IAPP and A $\beta 42$ peptides. Large oligomers and fibrillar species tumble slowly in solution on the NMR time-scale and are expected to give rise to signals that are too broadened to be directly observed by conventional liquid state NMR experiments. One-dimensional ${ }^{1} \mathrm{H}$ NMR spectra were recorded at $30{ }^{\circ} \mathrm{C}$ with a peptide concentration of $75 \mu \mathrm{M}$, under similar experimental conditions as for fluorescence, $\mathrm{CD}$ and TEM. Fig. 5 shows aliphatic methyl regions of $1 \mathrm{D}^{1} \mathrm{H}$ spectra and the integration of corresponding ${ }^{1} \mathrm{H}$ signals as a function of time. ${ }^{1} \mathrm{H}$ spectra acquired at initial times exhibit intense and narrow peaks, as expected for monomeric peptides of 37 and 42 residues. A rapid decay of signal intensity is observed for IAPP with a complete loss of signal in less than 3 hours (Fig. 5A and $\mathrm{B})$. In comparison, $\mathrm{A} \beta 42$ signals decay much more slowly and do not disappear completely even after 60 hours, the signal reaching a plateau corresponding to $25 \%$ of the peptide remaining in the monomeric form (Fig. 5B). Noteworthily, for both amyloid peptides, the decay of monomer population starts from the very beginning of the kinetics, with no apparent lag phase (within the time resolution of 1D experiment recording, which was about $20 \mathrm{~min}$ ). These experiments were repeated with peptide samples of different sources and showed similar behaviours regarding the decay of NMR signal, albeit with different kinetics and residual values for the monomer in the range 10 to $25 \%$. The kinetics of $A \beta 42$ consumption measured by NMR tend to be slower than that observed by fluorescence, which can be ascribed to interface effects with the container well and air that are different between NMR tubes and microtiter plates. ${ }^{50}$ Furthermore the use of $\mathrm{D}_{2} \mathrm{O}$ solvent instead of $\mathrm{H}_{2} \mathrm{O}$ was also shown to slightly affect the kinetics of aggregation,
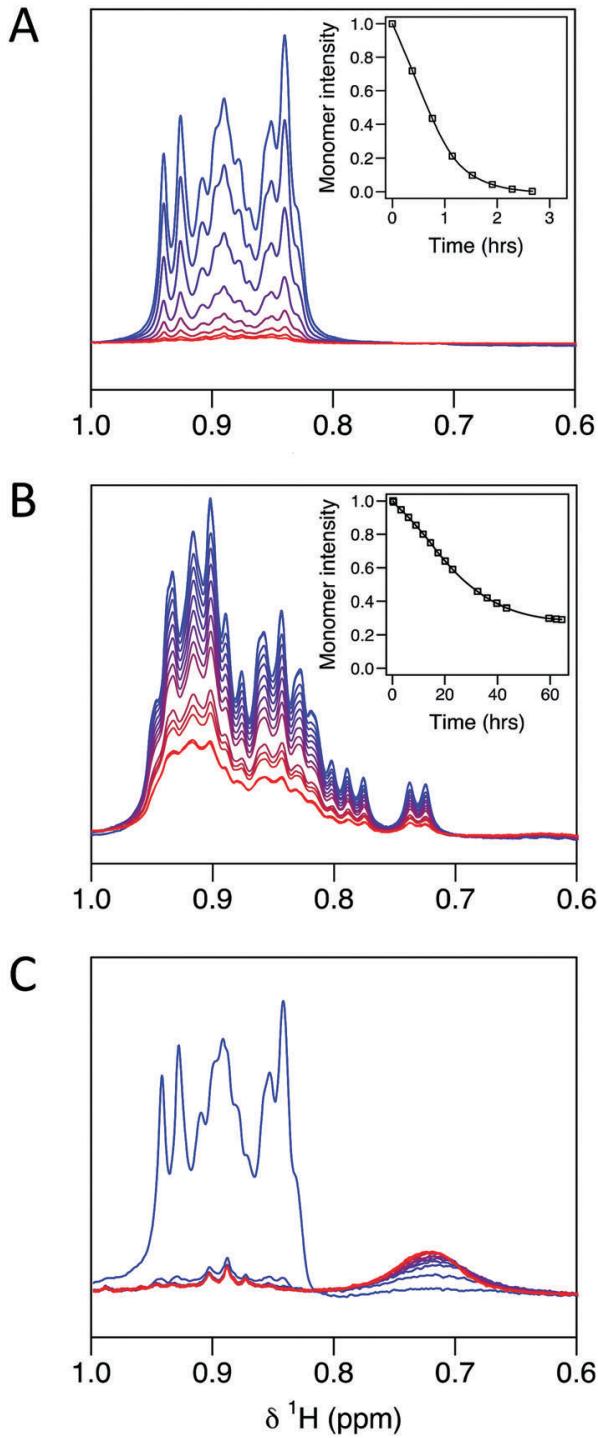

Fig. 5 Kinetics of IAPP (A and C) and A 42 (B) monomer consumption measured by $1 \mathrm{D}{ }^{1} \mathrm{H}$ NMR spectroscopy $\left(30{ }^{\circ} \mathrm{C}\right.$, peptide concentration of $75 \mu \mathrm{M}$ ). (A and B) Zoom in the methyl region (1-0.6 ppm) of $1 \mathrm{D}^{1} \mathrm{H}$ NMR spectra of IAPP (A) and A 42 (B) over time. The decay of monomer intensity, measured by integration over the aromatic and methyl regions, is shown in the insets. (C) Alternative behaviour observed for some IAPP samples showing the appearance of a broad upfield signal.

in the case of $\mathrm{A} \beta \mathrm{S}^{51}$ Interestingly, we observed for some but not all IAPP samples that, in parallel with the decrease of monomer signals, very broad signals appeared in the upfield region of ${ }^{1} \mathrm{H}$ spectra around 0.7 and $1.1 \mathrm{ppm}$ and gradually increased in intensity (Fig. 5C). These upfield signals probably originate from aggregated IAPP species of high molecular weight, which accumulate on time. However, as this contribution was not systematically observed for all prepared samples, it is likely that this NMR visible form represents an off-pathway species, rather than an intermediate on the monomer to fibril pathway. Other groups also reported similar observations. ${ }^{32}$ In the case of $A \beta 42$, we were not able to detect such broad signals in NMR samples. 

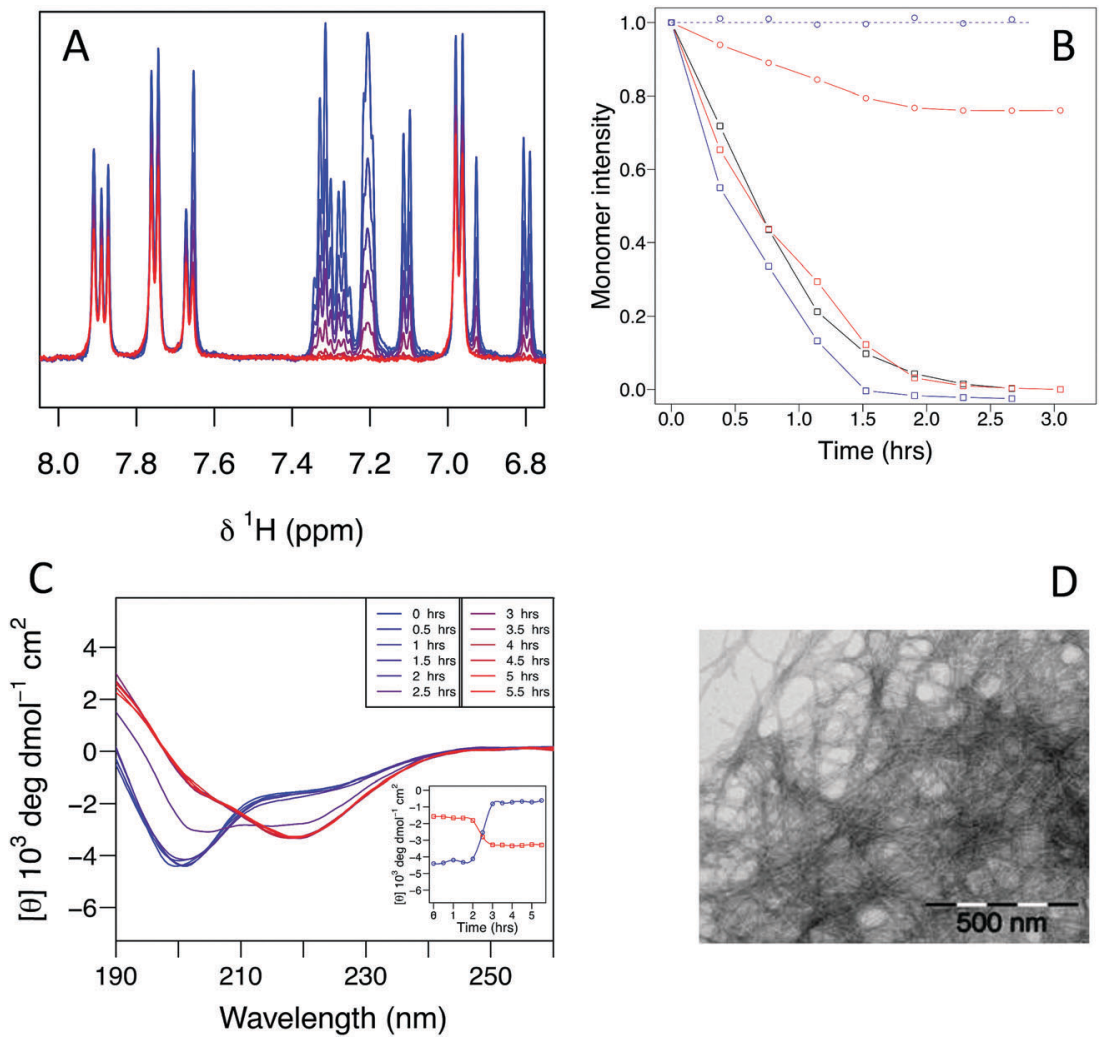

Fig. 6 Influence of ThT and TROL probes on IAPP fibril formation. (A) Aromatic region of 1D ${ }^{1} \mathrm{H}$ NMR spectra of IAPP in the presence of ThT recorded from initial time (blue) to 3 hour incubation (red) at $30{ }^{\circ} \mathrm{C}$. Signals of both IAPP and ThT probe are visible. (B) Time variation of ${ }^{1} \mathrm{H}$ resonance intensity for IAPP and fluorescent probes. The decay of ${ }^{1} \mathrm{H}$ signals of IAPP in the absence (black) or in the presence of ThT (red) or TROL (blue) is shown with squares. The evolution of ThT and signals is indicated with red and blue circles, respectively. (C) CD spectra of IAPP in the presence of ThT recorded at $30{ }^{\circ} \mathrm{C}$ every 30 min over 5.5 hours. The inset shows the time evolution of mean residue ellipticities taken at $200 \mathrm{~nm}$ (blue) and $218 \mathrm{~nm}$ (red). (D) TEM image of IAPP fibrils in the presence of ThT after 3 days.

An important condition to allow comparison of different fluorescent techniques is to evaluate potential effect of probe on the kinetics of amyloid fibril formation. Therefore, we took advantage of $1 \mathrm{D}^{1} \mathrm{H}$ NMR spectroscopy to analyze whether ThT or TROL could interfere with IAPP aggregation pathway. We observed that $1 \mathrm{D}^{1} \mathrm{H}$ signals decrease over time at similar rates in the absence and in the presence of an equimolar amount of ThT or TROL (Fig. 6A and B), indicating that these probes have no significant effect on the kinetics of depletion of the IAPP monomer. In addition, the inspection of the aromatic region (Fig. 6A and B) reveals that ThT signal decreases to $80 \%$ of its initial value, indicating that $20 \%$ of ThT gets bound to high molecular weight, NMR-invisible aggregated species. TROL, in contrast, does not show variation in signal intensity, suggesting that it does not incorporate into large NMR-invisible species. CD spectra kinetics also shows that ThT (Fig. 6C) and TROL (Fig. S1, ESI $\dagger$ ) have little influence on the random coil to $\beta$-sheet conformational transition, in agreement with similar morphology of final IAPP fibrils as observed by TEM (Fig. 6D and Fig. S1, ESI $\dagger$ ).

\subsection{Pulsed field gradient NMR experiments reveal different behaviours of $A \beta$ and IAPP during fibrillization}

The measurement of translational diffusion coefficients can provide useful information about the hydrodynamic properties of molecules. Diffusion coefficients depend on the size, shape and degree of compaction of peptides in solution and are also very sensitive to self-association equilibria. We measured by pulsed field gradient (PFG) NMR experiments the diffusion coefficients of IAPP and $\mathrm{A} \beta 42$ at a temperature of $30{ }^{\circ} \mathrm{C}$, on freshly prepared samples (Table 1 ). The values of hydrodynamic radius $R_{\mathrm{H}}$, inferred from Stokes-Einstein equation, are 14.8 and $12.1 \AA$ for IAPP and A $\beta 42$, respectively (Table 1), which are compatible with sizes expected for monomeric states. The higher value obtained for IAPP, although 5 residues shorter in length, may be ascribed to a less compact shape than $\mathrm{A} \beta 42$. The $R_{\mathrm{H}}$ value

Table 1 Hydrodynamic parameters (diffusion coefficient $D_{\mathrm{t}}$ and hydrodynamic radius $R_{\mathrm{H}}$ ) of IAPP and Aß42 measured by PFG NMR

\begin{tabular}{llllll}
\hline & \multicolumn{3}{c}{ Initial time $(0.6 \mathrm{~h})$} & \multicolumn{3}{c}{ After incubation period } \\
\cline { 2 - 3 }$T\left({ }^{\circ} \mathrm{C}\right)$ & $D_{\mathrm{t}}\left(10^{-10} \mathrm{~m}^{2} \mathrm{~s}^{-1}\right)$ & $R_{\mathrm{H}}(\AA)$ & $\begin{array}{l}\text { Time } \\
(\mathrm{h})\end{array}$ & $\begin{array}{l}\text { Monomer } \\
\text { population }(\%)\end{array}$ & $\begin{array}{l}D_{\mathrm{t}} \\
\left(10^{-10} \mathrm{~m}^{2} \mathrm{~s}^{-1}\right)\end{array}$ \\
\hline $\mathrm{A} \beta 42$ & & & & & \\
30 & $1.87 \pm 0.01$ & 12.1 & 20 & 64 & $1.49 \pm 0.01$ \\
$\mathrm{IAPP}$ & & & & & \\
30 & $1.52 \pm 0.01$ & 14.9 & 3 & 0 & n.d. \\
20 & $1.12 \pm 0.03$ & 15.3 & 3 & 22 & n.d. \\
15 & $0.97 \pm 0.01$ & 15.4 & 84 & 76 & $0.98 \pm 0.01$ \\
10 & $0.79 \pm 0.01$ & 15.6 & 50 & 93 & $0.80 \pm 0.01$
\end{tabular}


of IAPP is comparable to those previously measured for rat IAPP at $37{ }^{\circ} \mathrm{C}(14.1 \AA)$ of for human IAPP at $4{ }^{\circ} \mathrm{C}(15.3 \AA) .{ }^{32}$ These data, together with the observed narrow linewidths of ${ }^{1} \mathrm{H}$ resonances, indicate that both IAPP and A $\beta 42$ are predominantly monomeric at initial times.

The slower aggregation kinetics of $A \beta 42$, with respect to IAPP, enabled us to measure the diffusion coefficient of $A \beta 42$ over the time course of fibrillization (Fig. 7). Interestingly, the diffusion coefficient of $\mathrm{A} \beta 42$ was shown to decrease over time, exhibiting a 25\% drop during the first 20 hours (Fig. 7 and Table 1), which clearly highlights the self-association properties of $\mathrm{A} \beta 42$. As ${ }^{1} \mathrm{H}$ signals intensity on $1 \mathrm{D}$ spectra concomitantly decrease without notable changes in linewidths, it can be concluded that the observed signals correspond to the monomeric form of $\mathrm{A} \beta$. Consequently, the change in the diffusion coefficient can be ascribed to an equilibrium between the NMR visible $\mathrm{A} \beta 42$ monomeric form and oligomeric states that are not visible on 1D ${ }^{1} \mathrm{H}$ NMR spectra. The observed decrease of diffusion coefficient values upon time means that these oligomeric forms become more populated and/or increase in size, as they might have polydisperse size distribution. Interestingly, the time evolution of the diffusion constant differs after 20 hours: it no longer decreases but slowly increases during the next 40 hours. A possible explanation is that the oligomers in equilibrium with A 342 monomer become less populated in so far as they incorporate into very large fibrillar states which become dominant upon the fibrillization pathway. Therefore we conclude that the observed time dependence of $\mathrm{A} \beta 42$ diffusion coefficient can be ascribed to a dynamic equilibrium between monomers and early oligomeric states, but not with final fibrillar aggregated states. The oligomeric species in equilibrium with the monomer could be heterogeneous in nature, with varying size during the aggregation process.

We next examined if such time dependence of diffusion coefficient could be detected for IAPP. However the fast fibrillization kinetics at $30{ }^{\circ} \mathrm{C}$ precluded accurate measurements in the available time range. Therefore experiments were carried out at lower temperatures to slow down the fibrillization process. The measurement of diffusion coefficients on freshly prepared IAPP samples at 10, 15 and $20{ }^{\circ} \mathrm{C}$ (Table 1) gives comparable values of

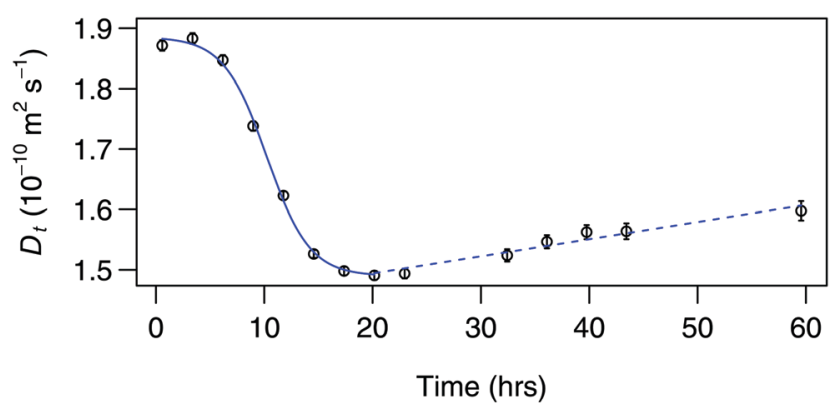

Fig. 7 Time dependence of the diffusion coefficient $D_{t}$ of $A \beta 42$ measured by PFG NMR. NMR experiments were measured at $30^{\circ} \mathrm{C}$ using a peptide concentration of $75 \mu \mathrm{M}$. The decrease of $D_{\mathrm{t}}$ in the first 20 hours was fitted to a Boltzmann equation and the subsequent increase in the 20-60 hours time lapse is shown as a hashed line. hydrodynamic radius $R_{\mathrm{H}}$ and therefore indicates similar size and degree of folding (or misfolding) in the $10-30{ }^{\circ} \mathrm{C}$ range. This contrasts with previous observations in literature, ${ }^{32}$ that reported a nearly 2 fold increase of the hydrodynamic radius upon lowering the temperature from 37 to $4{ }^{\circ} \mathrm{C}$, suggesting an important increase of peptide compactness upon cooling. In our hands, the limited variation of diffusion coefficients in the $10-30{ }^{\circ} \mathrm{C}$ temperature range does not support the presence of a temperaturedependent conformational equilibrium affecting the peptide compactness. One possible explanation to this discrepancy could be that the diffusion coefficients measured by Soong et al. ${ }^{32}$ might have been overestimated at high temperature owing to the presence of convection currents in NMR tubes. ${ }^{52}$ In our case, the use of Shigemi tubes probably circumvents such potential source of experimental error.

The fibrillization kinetics at $20{ }^{\circ} \mathrm{C}$ was still too fast to measure the diffusion coefficient on time. Lowering the temperature to $15{ }^{\circ} \mathrm{C}$ enabled to significantly slow down the fibrillization kinetics and to observe the monomer consumption over 4 days. Under these conditions, the measured diffusion coefficient remained constant throughout the kinetics (Table 1). Therefore no equilibrium between IAPP monomer and aggregated forms could be detected by PFG NMR experiments, in contrast with $\mathrm{A} \beta 42$. This underlines that oligomeric species do not likely contribute to measured diffusion coefficient, which therefore represents the monomeric state only. Different explanations could account for the absence of observation of such equilibrium. First, the population of these oligomeric species could be too weak to contribute to the observed average diffusion coefficient. Another possibility would be that the exchange rate is too slow and/or that the monomer to oligomer conversion is quasi irreversible.

The very broad upfield components that appear on some $1 \mathrm{D}{ }^{1} \mathrm{H}$ spectra during IAPP aggregation (Fig. 5C) correspond to species that virtually do not diffuse during the experimental diffusion delay (0.15 s) of PFG experiments. Indeed, the signal intensities show almost no attenuation upon pulsed field gradient application. This indicates that the diffusion coefficient of these species is smaller than at least two orders of magnitude, corresponding to an estimated hydrodynamic radius larger than $150 \mathrm{~nm}$. This confirms that these species correspond to large aggregates and not small oligomers.

\subsection{Saturation transfer difference experiments do not show the accumulation of oligomeric intermediates for IAPP, in contrast with $\mathbf{A \beta 4 2}$}

Finally, we used NMR magnetization transfer experiments in order to detect putative self-association equilibria between the monomeric peptide and higher molecular weight forms. ${ }^{53-55}$ Saturation transfer difference (STD) experiments consist in selective irradiation of the upfield $1 \mathrm{D}{ }^{1} \mathrm{H}$ region $(-0.5 \mathrm{ppm})$ which is devoid of signals from monomers but presumably contains broad, undetectable signals originating from higher molecular weight species in solution. If fast exchange conversion occurs between such species and monomers, saturation can then be transferred from the large unobserved oligomers to 
the monomer, leading to an attenuation of signal intensity of the NMR visible monomeric species. STD technique is expected to be more sensitive than PFG diffusion experiment to detect equilibria between monomer and aggregated species as even low populations $(1 \%)$ of aggregates can yield efficient saturation transfer to monomer form.

We recorded $1 \mathrm{D}{ }^{1} \mathrm{H}$ STD spectra for IAPP and A $\beta 42$ peptides and followed the time course of STD signals (Fig. 8). The STD signal was measured on the aromatic protons of both peptides to minimize the contribution of direct intramolecular magnetization transfer due to insufficient lack of selectivity of saturation pulses (applied at -0.5 and $30 \mathrm{ppm}$ for on- and off-resonance, respectively). For both peptides, virtually no STD signal is observed at initial times of incubation (within the first hour). Interestingly, in the case of A $\beta 42$, the STD signal was shown to gradually increase over time, with a linear variation over $60 \mathrm{~h}$ (Fig. 8B), proving that $\mathrm{A} \beta 42$ is in equilibrium with oligomeric species which become more populated with time and/or increase in size, leading to higher magnetization transfer efficiency. In order to get more insight on the species involved in saturation transfer, the experiments were carried out in the presence of preformed $\mathrm{A} \beta 42$ seeds to bypass the primary nucleation pathway. The addition of $10 \% \mathrm{~A} \beta 42$ seeds accelerates the kinetics of consumption of monomeric $\mathrm{A} \beta 42$, as expected, but does not influence the time course of STD increase (Fig. 8B). Importantly, the addition of seeds does not create an initial burst of the STD response, indicating that added aggregates are not directly involved in saturation transfer. Surprisingly, the STD increase does not seem to be affected by the differences of monomer consumption rates in the seeded and non-seeded experiments.

In order to be able to detect any evolution of STD over time, STD experiments were recorded for IAPP at lower temperatures of 10 (Fig. 8A) and $15{ }^{\circ} \mathrm{C}$ (data not shown). No significant STD signal was detected for IAPP, in contrast with A $\beta 42$, both at initial time and during the kinetics of monomer consumption. Thus, we observed no accumulation of high molecular weight species contributing to saturation transfer with monomers in the case of IAPP. As a control, STD experiments were also carried out on $\mathrm{A} \beta 42$ at low temperature (Fig. S2, ESI $\dagger$ ). A decrease of saturation transfer is observed upon cooling down from $30{ }^{\circ} \mathrm{C}$ to $10{ }^{\circ} \mathrm{C}$ but STD is still observed at these low temperatures.

\subsection{Gel electrophoresis fails to detect low molecular weight oligomeric species for IAPP}

In an attempt to directly visualize oligomeric species, Trisglycine gel electrophoresis experiments were conducted using IAPP and $A \beta_{42}$ peptide samples incubated at different times. A band corresponding to a single IAPP species is observed at initial time and progressively decreases in intensity during the first two hours of incubation (Fig. S3A, ESI $\dagger$ ). This result is in agreement with the kinetics of monomer depletion as followed by NMR. No additional band corresponding to higher molecular weight oligomeric species can be detected at any time of incubation. Altogether gel electrophoresis demonstrates that the monomer is fully consumed during the oligomerization process, but fails to detect low molecular weight IAPP forms. Similar PAGE experiments conducted on A $\beta 42$ samples (Fig. S3B, ESI $\dagger$ ) show the presence of small oligomeric species after 2 hours of incubation. However these results must be analysed with caution because it has been demonstrated that the presence of SDS in samples can induce artefactual oligomerisation in the case of $A \beta 42,{ }^{56-58}$ or oppositely it could dissociate oligomers. We conclude that in the case of IAPP, neither SDS-induced nor SDSresistant oligomers can be detected by this technique.

\section{Discussion}

Our results show that IAPP and A $\beta 42$ share some common features in the fibril formation processes, namely the overall profile of ThT fluorescence assays, the random coil to $\beta$ sheet CD transition and the morphology of amyloid fibrils. Nevertheless, these amyloid peptides exhibit marked differences regarding kinetics experiments, the detection of oligomers, and the observation of equilibria between monomer and oligomeric species, as discussed below.

We observed that the ThT fluorescence kinetics of IAPP fibril formation has a stronger autocatalytic behaviour than $\mathrm{A} \beta 42$
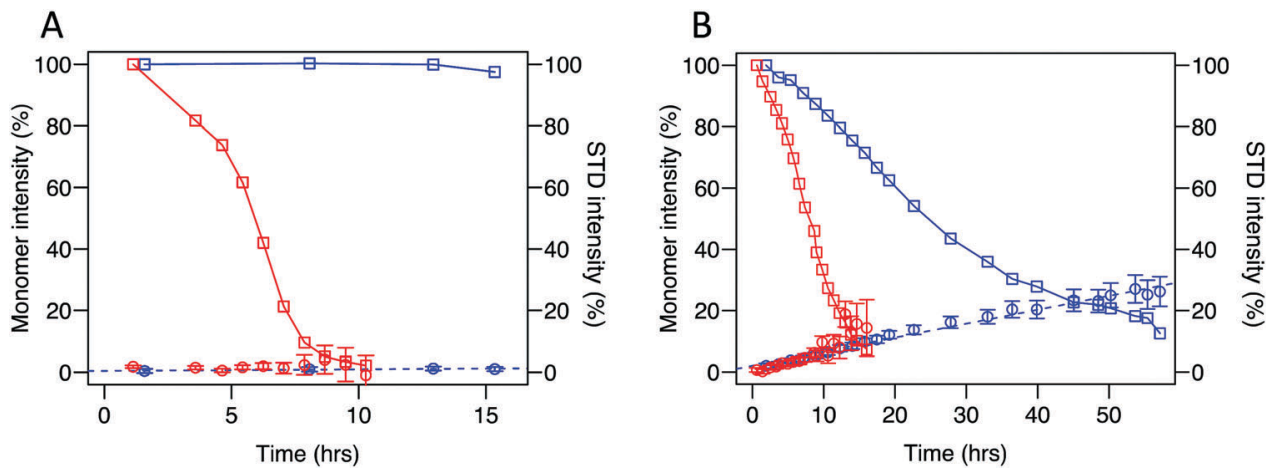

Fig. 8 Time variation of monomer population and STD signal intensity for IAPP (A) and AB42 (B), in the absence or in the presence of seeds. Intensities of $1 \mathrm{D}^{1} \mathrm{H}$ aromatic resonances and STD spectra are indicated by squares and circles, respectively. Experiments in the absence or in the presence of seeds are shown in blue and red, respectively. Experiments were recorded at $10{ }^{\circ} \mathrm{C}$ for IAPP and $30{ }^{\circ} \mathrm{C}$ for A $\beta 42$. STD intensity corresponds to the ratio $\left(I_{\text {off }}-I_{\text {on }}\right) / I_{\text {off }}$ where $I_{\text {off }}$ and $I_{\text {on }}$ are the intensities of aromatic signals in off-resonance and on-resonance saturation spectra, respectively. 
under the same experimental conditions, which suggests an involvement of secondary nucleation processes. Based on these results, we examined the concentration dependence of kinetic parameters to get information on the contribution of nucleation processes to fibril polymerization. Nucleation-dependent polymerization processes are expected to show a very high concentration dependence. In nucleation models, $t_{1 / 2}$ shows a concentration dependence as a power function $t_{1 / 2} \sim c^{\gamma}$. The exponent $\gamma$ depends on the size of the nucleus species as well as the type of dominant nucleation pathway, primary or secondary (i.e. fibril-catalysed). Primary nucleation-dependent processes typically require a $\gamma$ exponent lower than -1 .

We observed in the case of IAPP that the half-time of fibril formation $t_{1 / 2}$ obeys a power law as a function of concentration $t_{1 / 2} \sim c^{\gamma}$ with a $\gamma$ exponent of -0.2 , which corresponds to a low concentration dependence. The kinetics of IAPP fibrillogenesis has previously been examined by another group. ${ }^{5}$ Under similar concentration conditions (5-50 $\mu \mathrm{M}$ range) and using intrinsic tyrosine anisotropy fluorescence, the authors found that the half-time was virtually independent of peptide concentration. The slight differences in measured $\gamma$, from 0 to -0.2 , might be ascribed to differences in peptide purification and sample preparation. In both cases, IAPP was obtained by solid-phase peptide synthesis but the solvent conditions of purification were different (use of guanidium chloride, $\mathrm{pH}$, salt concentration).

Several models have been proposed to account for the weak dependence, or even independence, on peptide concentration. In the micelle model, the peptide is in rapid equilibrium with off-pathway micellar aggregates that buffer the monomer concentration, leading to an apparent low concentration dependence. Such formation of micellar species was indeed detected by NMR for $A \beta 42 .{ }^{59}$ In this case, the NMR signal intensity of monomer was found to increase during the initial incubation period, as well as the observed diffusion coefficient, proving the existence of transient fast-exchanging micellar species. In the case of IAPP, our NMR parameters did not follow such behaviour, indicating that IAPP is not in equilibrium with a micelle reservoir. An alternative model based on a supercritical concentration has been proposed to explain a weakening of concentration dependence. In this model, the monomer becomes less stable than oligomers beyond a supercritical concentration so that the peptide population becomes dominated by oligomeric species. However we found that even at high concentration, the NMR signal corresponds to a monomeric state of IAPP and no oligomers could be observed. This result is in agreement with recent $2 \mathrm{D}$ IR based studies which estimated the supercritical concentration of IAPP to be around $150-250 \mu \mathrm{M} .{ }^{36}$ So neither the micelle reservoir nor a supercritical regime are consistent with our NMR observations. Therefore the very low concentration dependence can be ascribed to saturable microscopic processes. A saturation of fibril elongation pathway can best account for the observed $\gamma$ value, which indicates that fibril elongation has a multistep nature.

The $\gamma$ value found for IAPP is higher than that observed for $A \beta 42$ under quiescent conditions $(-1.3)$ and also for $A \beta 40$ $(-1.2$ at low concentrations). Interestingly, $A \beta 40$ was characterized by a concentration-dependent $\gamma$, increasing from -1.2 at low concentrations to -0.2 at high concentrations. ${ }^{10}$ This more complex behaviour of $\mathrm{A} \beta 40$ was ascribed to an increased contribution of fibril-catalyzed secondary pathways, which become saturable at high concentrations. It is possible that secondary pathways at the surface of fibrils may also become saturable in the case of IAPP.

Although not directly observable, the presence of oligomers can possibly be detected by NMR spectroscopy through the characterisation of dynamic equilibria between monomers and oligomers. Indeed, our NMR study applied to A $\beta 42$ was successful in detecting the presence of oligomeric species with which the monomeric form interconverts. The decrease of the measured diffusion coefficient during the incubation period supports the accumulation of a fast-exchanging oligomeric species. Saturation transfer efficiency was also shown to increase over the whole incubation period, indicating the presence of higher molecular weight species that accumulate, and possibly increase in size. The continuous build-up of STD suggests that the involved NMRinvisible species are large aggregates that accumulate on time, rather than lower molecular weight nucleated species that would occur transiently prior to polymer elongation. Seeding experiments indicate that aggregated seeds are not the involved species. The physical basis of the detected equilibrium could be a reversible incorporation into large aggregated species or transient contacts with the surface of aggregated species. Interestingly, a dynamic equilibrium with prefibrillar aggregates was previously characterized for $A \beta 40$ and $A \beta 42$ using a different approach based on ${ }^{15} \mathrm{~N}$ relaxation measurements and ${ }^{15} \mathrm{~N}$ saturation transfer experiments. ${ }^{60,61}$

In contrast, in the case of IAPP, neither PFG diffusion nor STD experiments could detect significant presence of oligomeric species. PFG measurements show a decrease of IAPP hydrodynamic radius with temperature, which can be best explained by a slightly increased compactness as a higher content in IAPP helical structure upon heating was previously observed. ${ }^{32}$ An intermolecular contribution to the measured diffusion coefficient appears to be unlikely as we did not observe any time dependence during polymerization. No build-up of STD signal is observed with time, in contrast with $\mathrm{A} \beta 42$, indicating that there is no accumulation of species with which the monomer interconverts. Several factors could explain our observations. First, the exchange rate between the monomer and NMR invisible species may be too low to allow efficient magnetization transfer. The fast fibrillization kinetics of IAPP compelled us to work below room temperature, which may be also detrimental to the regime of exchanging rates compatible with saturation transfer. Actually we observed a decrease of saturation transfer efficiency at low temperature in the case of A $\beta 42$, but STD was still detectable at the lowest investigated temperatures. Another possibility is that the oligomeric species may have very low concentration and/or short lifetime. The weak population of oligomeric species is in agreement with our results and other studies based on $1 \mathrm{D}{ }^{1} \mathrm{H}$ and ${ }^{19} \mathrm{~F} \mathrm{NMR}^{32,33}$ and ultracentrifugation. ${ }^{31}$ One of the technique that could detect small oligomeric species (dimer to hexamers) is IMS-MS ${ }^{34}$ but it is difficult 
to estimate the population of such species and evaluate their on-pathway or off-pathway relationships. Finally, the observation of $\beta$-sheet oligomers by $2 \mathrm{D}$ infrared spectroscopy ${ }^{35,36}$ was only possible above the supercritical concentration, requiring highly concentrated samples (about $0.5 \mathrm{mM}$ ).

While amyloid peptides present common features in fibril formation, our study shows that IAPP and A $\beta 42$ exhibit significant differences concerning microscopic events of amyloid species formation. The lack of detection of oligomers in the lag phase of IAPP fibril formation suggests that these species are too unstable to give rise to significant populations. The absence of build-up of early oligomers in the case of IAPP hampers the structural characterization of such transient species and the rational design of selective inhibitors.

\section{Conflicts of interest}

There are no conflicts to declare.

\section{Acknowledgements}

We acknowledge Christophe Piesse (IFR83-Université Pierre et Marie Curie, France) for the synthesis of amyloid peptides and Jean-Jacques Lacapère for electron microscopy.

\section{References}

1 F. Chiti and C. M. Dobson, Protein Misfolding, Functional Amyloid, and Human Disease, Annu. Rev. Biochem., 2006, 75, 333-366.

2 T. P. J. Knowles, M. Vendruscolo and C. M. Dobson, The amyloid state and its association with protein misfolding diseases, Nat. Rev. Mol. Cell Biol., 2014, 15, 384-396.

3 J. W. Höppener, B. Ahrén and C. J. Lips, Islet amyloid and type 2 diabetes mellitus, N. Engl. J. Med., 2000, 343, 411-419.

4 P. Westermark, A. Andersson and G. T. Westermark, Islet Amyloid Polypeptide, Islet Amyloid, and Diabetes Mellitus, Physiol. Rev., 2011, 91, 795-826.

5 S. B. Padrick and A. D. Miranker, Islet Amyloid: Phase Partitioning and Secondary Nucleation Are Central to the Mechanism of Fibrillogenesis, Biochemistry, 2002, 41, 4694-4703.

6 L. Caillon, A. R. F. Hoffmann, A. Botz and L. Khemtemourian, Molecular Structure, Membrane Interactions, and Toxicity of the Islet Amyloid Polypeptide in Type 2 Diabetes Mellitus, J. Diabetes Res., 2016, 2016, 1-13.

7 P. Arosio, T. P. J. Knowles and S. Linse, On the lag phase in amyloid fibril formation, Phys. Chem. Chem. Phys., 2015, 17, 7606-7618.

8 E. Hellstrand, B. Boland, D. M. Walsh and S. Linse, Amyloid $\beta$-Protein Aggregation Produces Highly Reproducible Kinetic Data and Occurs by a Two-Phase Process, ACS Chem. Neurosci., 2010, 1, 13-18.

9 S. I. A. Cohen, S. Linse, L. M. Luheshi, E. Hellstrand, D. A. White, L. Rajah, D. E. Otzen, M. Vendruscolo, C. M. Dobson and T. P. J. Knowles, Proliferation of amyloid-42 aggregates occurs through a secondary nucleation mechanism, Proc. Natl. Acad. Sci. U. S. A., 2013, 110, 9758-9763.

10 G. Meisl, X. Yang, E. Hellstrand, B. Frohm, J. B. Kirkegaard, S. I. A. Cohen, C. M. Dobson, S. Linse and T. P. J. Knowles, Differences in nucleation behavior underlie the contrasting aggregation kinetics of the $\mathrm{A} \beta 40$ and $\mathrm{A} \beta 42$ peptides, Proc. Natl. Acad. Sci. U. S. A., 2014, 111, 9384-9389.

11 R. Kayed, E. Head, J. L. Thompson, T. M. McIntire, S. C. Milton, C. W. Cotman and C. G. Glabe, Common structure of soluble amyloid oligomers implies common mechanism of pathogenesis, Science, 2003, 300, 486-489.

12 L. Nagel-Steger, M. C. Owen and B. Strodel, An account of Amyloid Oligomers: Facts and Figures Obtained from Experiments and Simulations, ChemBioChem, 2016, 17, 657-676.

13 M. Stefani, Structural features and cytotoxicity of amyloid oligomers: implications in Alzheimer's disease and other diseases with amyloid deposits, Prog. Neurobiol., 2012, 99, 226-245.

14 J. J. Meier, R. Kayed, C.-Y. Lin, T. Gurlo, L. Haataja, S. Jayasinghe, R. Langen, C. G. Glabe and P. C. Butler, Inhibition of human IAPP fibril formation does not prevent beta-cell death: evidence for distinct actions of oligomers and fibrils of human IAPP, Am. J. Physiol.: Endocrinol. Metab., 2006, 291, E1317-E1324.

15 L. Haataja, T. Gurlo, C. J. Huang and P. C. Butler, Islet Amyloid in Type 2 Diabetes, and the Toxic Oligomer Hypothesis, Endocr. Rev., 2008, 29, 303-316.

16 M. F. M. Engel, L. Khemtemourian, C. C. Kleijer, H. J. D. Meeldijk, J. Jacobs, A. J. Verkleij, B. de Kruijff, J. A. Killian and J. W. M. Hoppener, Membrane damage by human islet amyloid polypeptide through fibril growth at the membrane, Proc. Natl. Acad. Sci. U. S. A., 2008, 105, 6033-6038.

17 A. Jan, O. Adolfsson, I. Allaman, A.-L. Buccarello, P. J. Magistretti, A. Pfeifer, A. Muhs and H. A. Lashuel, A $\beta 42$ Neurotoxicity Is Mediated by Ongoing Nucleated Polymerization Process Rather than by Discrete A $\beta 42$ Species, J. Biol. Chem., 2011, 286, 8585-8596.

18 J. Lee, E. K. Culyba, E. T. Powers and J. W. Kelly, Amyloid- $\beta$ forms fibrils by nucleated conformational conversion of oligomers, Nat. Chem. Biol., 2011, 7, 602-609.

19 I. Benilova, E. Karran and B. De Strooper, The toxic A $\beta$ oligomer and Alzheimer's disease: an emperor in need of clothes, Nat. Neurosci., 2012, 15, 349-357.

20 B. Soreghan, J. Kosmoski and C. Glabe, Surfactant properties of Alzheimer's A beta peptides and the mechanism of amyloid aggregation, J. Biol. Chem., 1994, 269, 28551-28554.

21 A. E. Roher, M. O. Chaney, Y. M. Kuo, S. D. Webster, W. B. Stine, L. J. Haverkamp, A. S. Woods, R. J. Cotter, J. M. Tuohy, G. A. Krafft, B. S. Bonnell and M. R. Emmerling, Morphology and toxicity of Abeta-(1-42) dimer derived from neuritic and vascular amyloid deposits of Alzheimer's disease, J. Biol. Chem., 1996, 271, 20631-20635.

22 G. M. Shankar, S. Li, T. H. Mehta, A. Garcia-Munoz, N. E. Shepardson, I. Smith, F. M. Brett, M. A. Farrell, M. J. Rowan, 
C. A. Lemere, C. M. Regan, D. M. Walsh, B. L. Sabatini and D. J. Selkoe, Amyloid- $\beta$ protein dimers isolated directly from Alzheimer's brains impair synaptic plasticity and memory, Nat. Med., 2008, 14, 837-842.

23 S. Lesné, M. T. Koh, L. Kotilinek, R. Kayed, C. G. Glabe, A. Yang, M. Gallagher and K. H. Ashe, A specific amyloid- $\beta$ protein assembly in the brain impairs memory, Nature, 2006, 440, 352-357.

24 R. Kayed, A. Pensalfini, L. Margol, Y. Sokolov, F. Sarsoza, E. Head, J. Hall and C. Glabe, Annular Protofibrils Are a Structurally and Functionally Distinct Type of Amyloid Oligomer, J. Biol. Chem., 2009, 284, 4230-4237.

25 M. K. Jana, R. Cappai, C. L. L. Pham and G. D. Ciccotosto, Membrane-bound tetramer and trimer $\mathrm{A} \beta$ oligomeric species correlate with toxicity towards cultured neurons, J. Neurochem., 2016, 136, 594-608.

26 L.-M. Yan, A. Velkova, M. Tatarek-Nossol, E. Andreetto and A. Kapurniotu, IAPP Mimic Blocks A $\beta$ Cytotoxic SelfAssembly: Cross-Suppression of Amyloid Toxicity of $\mathrm{A} \beta$ and IAPP Suggests a Molecular Link between Alzheimer's Disease and Type II Diabetes, Angew. Chem., Int. Ed., 2007, 46, 1246-1252.

27 R. Hu, M. Zhang, H. Chen, B. Jiang and J. Zheng, CrossSeeding Interaction between $\beta$-Amyloid and Human Islet Amyloid Polypeptide, ACS Chem. Neurosci., 2015, 6, 1759-1768.

28 A. Demuro, E. Mina, R. Kayed, S. C. Milton, I. Parker and C. G. Glabe, Calcium Dysregulation and Membrane Disruption as a Ubiquitous Neurotoxic Mechanism of Soluble Amyloid Oligomers, J. Biol. Chem., 2005, 280, 17294-17300.

29 J. Janson, R. H. Ashley, D. Harrison, S. McIntyre and P. C. Butler, The mechanism of islet amyloid polypeptide toxicity is membrane disruption by intermediate-sized toxic amyloid particles, Diabetes, 1999, 48, 491-498.

30 H.-L. Zhao, Y. Sui, J. Guan, L. He, X.-M. Gu, H. K. Wong, L. Baum, F. M. M. Lai, P. C. Y. Tong and J. C. N. Chan, Amyloid oligomers in diabetic and nondiabetic human pancreas, Transl. Res., 2009, 153, 24-32.

31 S. M. Vaiana, R. Ghirlando, W.-M. Yau, W. A. Eaton and J. Hofrichter, Sedimentation Studies on Human Amylin Fail to Detect Low-Molecular-Weight Oligomers, Biophys. J., 2008, 94, L45-L47.

32 R. Soong, J. R. Brender, P. M. Macdonald and A. Ramamoorthy, Association of Highly Compact Type II Diabetes Related Islet Amyloid Polypeptide Intermediate Species at Physiological Temperature Revealed by Diffusion NMR Spectroscopy, J. Am. Chem. Soc., 2009, 131, 7079-7085.

33 Y. Suzuki, J. R. Brender, K. Hartman, A. Ramamoorthy and E. N. G. Marsh, Alternative Pathways of Human Islet Amyloid Polypeptide Aggregation Distinguished by ${ }^{19} \mathrm{~F}$ Nuclear Magnetic Resonance-Detected Kinetics of Monomer Consumption, Biochemistry, 2012, 51, 8154-8162.

34 L. M. Young, P. Cao, D. P. Raleigh, A. E. Ashcroft and S. E. Radford, Ion Mobility Spectrometry-Mass Spectrometry Defines the Oligomeric Intermediates in Amylin Amyloid Formation and the Mode of Action of Inhibitors, J. Am. Chem. Soc., 2014, 136, 660-670.
35 L. E. Buchanan, E. B. Dunkelberger, H. Q. Tran, P.-N. Cheng, C.-C. Chiu, P. Cao, D. P. Raleigh, J. J. de Pablo, J. S. Nowick and M. T. Zanni, Mechanism of IAPP amyloid fibril formation involves an intermediate with a transient ß-sheet, Proc. Natl. Acad. Sci. U. S. A., 2013, 110, 19285-19290.

36 A. L. Serrano, J. P. Lomont, L.-H. Tu, D. P. Raleigh and M. T. Zanni, A Free Energy Barrier Caused by the Refolding of an Oligomeric Intermediate Controls the Lag Time of Amyloid Formation by hIAPP, J. Am. Chem. Soc., 2017, 139, 16748-16758.

37 L. Khemtémourian, M. F. M. Engel, J. A. W. Kruijtzer, J. W. M. Höppener, R. M. J. Liskamp and J. A. Killian, The role of the disulfide bond in the interaction of islet amyloid polypeptide with membranes, Eur. Biophys. J., 2010, 39, 1359-1364.

38 L. Caillon, O. Lequin and L. Khemtémourian, Evaluation of membrane models and their composition for islet amyloid polypeptide-membrane aggregation, Biochim. Biophys. Acta, 2013, 1828, 2091-2098.

39 J. Kaffy, D. Brinet, J.-L. Soulier, I. Correia, N. Tonali, K. F. Fera, Y. Iacone, A. R. F. Hoffmann, L. Khemtemourian, B. Crousse, M. Taylor, D. Allsop, M. Taverna, O. Lequin and S. Ongeri, Designed Glycopeptidomimetics Disrupt Protein-Protein Interactions Mediating Amyloid $\beta$-Peptide Aggregation and Restore Neuroblastoma Cell Viability, J. Med. Chem., 2016, 59, 2025-2040.

40 T. M. Ryan, J. Caine, H. D. T. Mertens, N. Kirby, J. Nigro, K. Breheney, L. J. Waddington, V. A. Streltsov, C. Curtain, C. L. Masters and B. R. Roberts, Ammonium hydroxide treatment of $A \beta$ produces an aggregate free solution suitable for biophysical and cell culture characterization, PeerJ, 2013, 1, e73.

41 L. Caillon, L. Duma, O. Lequin and L. Khemtemourian, Cholesterol modulates the interaction of the islet amyloid polypeptide with membranes, Mol. Membr. Biol., 2014, 31, 239-249.

42 W. S. Price, Pulsed-field gradient nuclear magnetic resonance as a tool for studying translational diffusion: part II. Experimental aspects, Concepts Magn. Reson., 1998, 10, 197-237.

43 R. Mills, Self-diffusion in normal and heavy water in the range 1-45 deg, J. Phys. Chem., 1973, 77, 685-688.

44 C. H. Cho, J. Urquidi, S. Singh and G. W. Robinson, Thermal Offset Viscosities of Liquid $\mathrm{H}_{2} \mathrm{O}, \mathrm{D}_{2} \mathrm{O}$, and $\mathrm{T}_{2} \mathrm{O}, J$. Phys. Chem. B, 1999, 103, 1991-1994.

45 B. Dorgeret, L. Khemtémourian, I. Correia, J.-L. Soulier, O. Lequin and S. Ongeri, Sugar-based peptidomimetics inhibit amyloid $\beta$-peptide aggregation, Eur. J. Med. Chem., 2011, 46, 5959-5969.

46 H. LeVine, Quantification of beta-sheet amyloid fibril structures with thioflavin T, Methods Enzymol., 1999, 309, 274-284.

47 A. A. Reinke and J. E. Gestwicki, Insight into Amyloid Structure Using Chemical Probes: Amyloid Structure Using Chemical Probes, Chem. Biol. Drug Des., 2011, 77, 399-411.

48 A. A. Reinke, G. A. Abulwerdi and J. E. Gestwicki, Quantifying Prefibrillar Amyloids in vitro by Using a 'Thioflavin-Like' Spectroscopic Method, ChemBioChem, 2010, 11, 1889-1895. 
49 E. T. Powers and D. L. Powers, The Kinetics of Nucleated Polymerizations at High Concentrations: Amyloid Fibril Formation Near and Above the 'Supercritical Concentration', Biophys. J., 2006, 91, 122-132.

50 L. Jean, C. F. Lee and D. J. Vaux, Enrichment of Amyloidogenesis at an Air-Water Interface, Biophys. J., 2012, 102, 1154-1162.

51 J. Roche, Y. Shen, J. H. Lee, J. Ying and A. Bax, Monomeric $\mathrm{A} \beta_{1-40}$ and $\mathrm{A} \beta_{1-42}$ Peptides in Solution Adopt Very Similar Ramachandran Map Distributions That Closely Resemble Random Coil, Biochemistry, 2016, 55, 762-775.

52 I. Swan, M. Reid, P. W. A. Howe, M. A. Connell, M. Nilsson, M. A. Moore and G. A. Morris, Sample convection in liquidstate NMR: why it is always with us, and what we can do about it, J. Magn. Reson., 2015, 252, 120-129.

53 A. Bhunia, S. Bhattacharjya and S. Chatterjee, Applications of saturation transfer difference NMR in biological systems, Drug Discovery Today, 2012, 17, 505-513.

54 S. Narayanan and B. Reif, Characterization of chemical exchange between soluble and aggregated states of betaamyloid by solution-state NMR upon variation of salt conditions, Biochemistry, 2005, 44, 1444-1452.

55 J. Milojevic, V. Esposito, R. Das and G. Melacini, Understanding the molecular basis for the inhibition of the Alzheimer's Abeta-peptide oligomerization by human serum albumin using saturation transfer difference and off-resonance relaxation NMR spectroscopy, J. Am. Chem. Soc., 2007, 129, 4282-4290.

56 G. Bitan, E. A. Fradinger, S. M. Spring and D. B. Teplow, Neurotoxic protein oligomers - what you see is not always what you get, Amyloid, 2005, 12, 88-95.

57 A. Sandberg, L. M. Luheshi, S. Sollvander, T. Pereira de Barros, B. Macao, T. P. J. Knowles, H. Biverstal, C. Lendel, F. Ekholm-Petterson, A. Dubnovitsky, L. Lannfelt, C. M. Dobson and T. Hard, Stabilization of neurotoxic Alzheimer amyloid-oligomers by protein engineering, Proc. Natl. Acad. Sci. U. S. A., 2010, 107, 15595-15600.

58 R. Pujol-Pina, S. Vilaprinyó-Pascual, R. Mazzucato, A. Arcella, M. Vilaseca, M. Orozco and N. Carulla, SDS-PAGE analysis of $\mathrm{A} \beta$ oligomers is disserving research into Alzheimer's disease: appealing for ESI-IM-MS, Sci. Rep., 2015, 5, 14809.

59 M. A. Wälti, J. Orts, B. Vögeli, S. Campioni and R. Riek, Solution NMR Studies of Recombinant A $\beta(1-42)$ : From the Presence of a Micellar Entity to Residual $\beta$-Sheet Structure in the Soluble Species, ChemBioChem, 2015, 16, 659-669.

60 N. L. Fawzi, J. Ying, D. A. Torchia and G. M. Clore, Kinetics of Amyloid $\beta$ Monomer-to-Oligomer Exchange by NMR Relaxation, J. Am. Chem. Soc., 2010, 132, 9948-9951.

61 N. L. Fawzi, J. Ying, R. Ghirlando, D. A. Torchia and G. M. Clore, Atomic-resolution dynamics on the surface of amyloid- $\beta$ protofibrils probed by solution NMR, Nature, 2011, 480, 268-272. 